\title{
Tuberculous bowel obstruction at a university teaching hospital in Northwestern Tanzania: a surgical experience with 118 cases
}

Phillipo L Chalya ${ }^{1 *}$, Mabula D Mchembe ${ }^{2 \dagger}$, Stephen E Mshana ${ }^{3 \dagger}$, Peter Rambau ${ }^{4 \dagger}$, Hyasinta Jaka ${ }^{5 \dagger}$ and Joseph B Mabula ${ }^{1+}$

\begin{abstract}
Background: Bowel obstruction resulting from intestinal tuberculosis has been reported to be more prevalent in developing countries including Tanzania. This study was undertaken to describe the clinicopathological profile, surgical management and outcome of tuberculous intestinal obstruction in our local setting and to identify factors responsible for poor outcome among these patients.

Methods: This was a prospective descriptive study of patients operated for tuberculous intestinal obstruction at Bugando Medical Centre (BMC) in northwestern Tanzania from April 2008 to March 2012. Ethical approval to conduct the study was obtained from relevant authorities. Statistical data analysis was performed using SPSS version 17.0.
\end{abstract}

Results: A total of 118 patients with tuberculous intestinal obstruction were studied. The male to female ratio was 1.8: 1. The median age was 26 years (range 11-67 years). The modal age group was 21-30 years. Thirty-one (26.3\%) patients had associated pulmonary tuberculosis and 25 (21.2\%) patients were HIV positive with a median CD4+ count of 225 cells $/ \mu \mathrm{l}$. Small bowel strictures were the most common operative findings accounting for $72.9 \%$ of cases. The ileo-caecal region was the commonest area of involvement in 68 (57.6\%) patients. The right hemicolectomy with ileo-transverse anastomosis was the most frequent surgical procedure performed in 66 (55.9\%) patients. Postoperatively all the patients received antituberculous drugs for a period of one year. Postoperative complication rate was $37.3 \%$ and surgical site infection (SSI) was the most frequent complication in $42.8 \%$ of cases. HIV positivity and low CD4+ count were the main predictors of SSI $(p<0.001)$. The overall median length of hospital stay was 24 days. Patients who had postoperative complications stayed longer in the hospital and this was statistically significant $(p=0.011)$. Mortality rate was $28.8 \%$ and it was significantly associated with co-existing medical illness, delayed presentation, HIV positivity, low CD 4 count $(<200$ cells/ $\mu$ l), ASA class and presence of complications $(p<0.001)$. The follow up of patients was generally poor as more than fifty percent of patients were lost to follow up.

Conclusion: Tuberculous bowel obstruction remains rampant in our environment and contributes significantly to high morbidity and mortality. The majority of patients present late when the disease becomes complicated. A high index of suspicion, proper evaluation and therapeutic trial in suspected patients is essential for an early diagnosis and timely definitive treatment, in order to decrease the morbidity and mortality associated with this disease.

Keywords: Bowel obstruction, Intestinal tuberculosis, Clinicopathological profile, Surgical management, Outcome, Tanzania

\footnotetext{
* Correspondence: drphillipoleo@yahoo.com

${ }^{\dagger}$ Equal contributors

'Department of Surgery, Catholic University of Health and Allied Sciences-

Bugando, Mwanza, Tanzania

Full list of author information is available at the end of the article
}

\section{Ciomed Central}

(c) 2013 Chalya et al.; licensee BioMed Central Ltd. This is an Open Access article distributed under the terms of the Creative Commons Attribution License (http://creativecommons.org/licenses/by/2.0), which permits unrestricted use, distribution, and reproduction in any medium, provided the original work is properly cited. 


\section{Background}

Tuberculosis (TB), a communicable disease caused by Mycobacterium tuberculosis, is a common and major health problem worldwide [1]. Approximately one third of the world population is infected and about three millions die each year from this disease [1,2]. In developed countries the incidence of TB has become rare due to increased standards of living [3]. However, due to the influx of immigrants from third world countries, HIV infection and increasing use of Immunosuppressive therapy, the incidence of tuberculosis in developed countries is again on the rise [4]. In developing countries, tuberculosis remains the principal cause of death, probably due to ignorance, poverty, overcrowding, poor sanitation, malnutrition and coexistence with emergent diseases like AIDS [5]. Approximately $95 \%$ of new cases and $98 \%$ of deaths occur in developing countries $[6,7]$.

Tuberculosis may involve any part of the body but abdomen is one of the commonest site of involvement after lungs [8]. In the abdomen, tuberculosis may affect the gastro-intestinal tract, peritoneum, lymph nodes and solid viscera.

The modes of infection of the intestinal tuberculosis include hematogenous spread from a primary lung focus that reactivates later or miliary tuberculosis, spread via lymphatics from infected nodes, ingestion of bacilli either from the sputum or from infected sources such as milk products, or by direct spread from adjacent organs [9].

Intestinal tuberculosis has usually one of the three main forms i.e. ulcerative, hypertrophic or ulcerohypertrophic, and fibrous stricturing form [10,11]. The disease can mimic various gastrointestinal disorders, particularly the inflammatory bowel disease, colonic malignancy, or other gastrointestinal infections [12].

It usually runs an indolent course and presents late with complications especially acute or sub-acute intestinal obstruction due to mass (tuberculoma) or stricture formation in small gut and ileocaecal region or gut perforation leading to peritonitis $[13,14]$. In spite of advances in medical imaging, the early diagnosis of abdominal tuberculosis is still a problem due to vague and non-specific symptoms and patients usually present when complications such as bowel obstruction or perforation had occurred [15]. The most common complication of abdominal tuberculosis is obstruction due to narrowing of the lumen by hyperplastic caecal tuberculosis, by strictures of the small intestine, which are commonly multiple, or by adhesions and emergency surgery has to be resorted for confirmation of the diagnosis or for relief of obstruction $[15,16]$.

The management of intestinal obstruction due to tuberculosis involves surgery and postoperative treatment with anti-tuberculous therapy [15,17]. The disease, though potentially curable and preventable, still carries a significant morbidity and mortality in Tanzania despite establishment of the National Tuberculosis and Leprosy Programme (NTLP) which was launched by the Ministry of Health and Social Welfare in 1977 as a single combined programme. Factors responsible for this state of affairs are not known. The incidence of tuberculosis has increased dramatically in the last two decades driven by the spread of HIV infection. This increase in incidence has dramatically increased the workload of health care providers and overstretched the existing health systems.

In recent years, our centre has observed a sudden increase in the number of patients with bowel obstruction secondary to intestinal tuberculosis. This observation prompted the authors to analyze this problem. The aim of this study was to describe our experiences in the management of bowel obstruction due to intestinal tuberculosis, outlining the clinicopathological profile, surgical management and outcome of tuberculous intestinal obstruction in our local setting and to identify factors responsible for poor outcome among these patients.

\section{Methods}

\section{Study design and setting}

This was a prospective descriptive study of patients operated for tuberculous intestinal obstruction at Bugando Medical Centre (BMC) in northwestern Tanzania from April 2008 to March 2012. BMC is located in Mwanza city along the shore of Lake Victoria in the northwestern part of Tanzania. It is a tertiary care and teaching hospital for the Catholic University of Health and Allied SciencesBugando (CUHAS-Bugando) and other paramedics and has a bed capacity of 1000 . BMC is one of the four largest referral hospitals in the country and serves as a referral centre for tertiary specialist care for a catchment population of approximately 13 million people.

\section{Study population}

All patients who were operated for intestinal obstruction at BMC during the period of study and in whom the operative and histopathological findings were suggestive of tuberculosis were consecutively enrolled into the study. Patients who failed to give proper history and those without next of kin to consent for the study were excluded from the study. Patients who failed to consent for HIV infection testing were also excluded from the study.

Preoperatively, all the patients recruited into the study had intravenous fluids to correct fluid and electrolyte deficits; nasogastric suction; urethral catheterization and broad-spectrum antibiotic coverage. Relevant preoperative investigations included packed cell volume, serum electrolytes, urea and creatinine, blood grouping and crossmatching and erythrocyte sedimentation rate (ESR). Patients were also screened for HIV testing using Tanzania HIV Rapid Test Algorithm [18] and CD 4+ count using FACS or FACSCALIBUR from BD Biosciences USA. A 
determination of CD 4 count was only performed in HIV positive patients. Radiological investigations including Xray abdomen erect and supine, $\mathrm{X}$-ray chest $\mathrm{PA}$-view were done in all patients. Abdominal ultrasound was also performed in some patients suspected to have associated abdominal collections. Patients presenting in a critical condition were treated with vital system support by: administration of Oxygen, ionotropic support when found hypotensive and oliguric despite adequate fluid replacement. After resuscitation, all patients, under general anesthesia were subjected to exploratory laparotomy through midline incision. They had pre-operative anesthetic assessment using the American Society of Anesthetists (ASA) classification [19] as shown in Table 1. To minimize variability in our study, the assignation of ASA class was performed by one consultant anesthetist adhering strictly to criteria above. Adequate hydration was indicated by an hourly urine output of $30 \mathrm{ml} /$ hour. The operations were performed either by a consultant surgeon or a senior resident under the direct supervision of a consultant surgeon.

Intraoperative tissue biopsy was taken for histopathological studies; a portion of the tissue was fixed in 10 per cent formalin; routine processing was done as per standard operative procedures and stained with haemotoxylin and eosin. Presence of caseating granulomas surrounded by epitheloid cells, lymphocytes, plasma cells and giant cells were diagnostic of tuberculosis [20,21]. Postoperatively patients were kept nil orally till return of bowl sounds and at that time nasogastric tubes were removed. Intravenous antibiotics were used for up to one week. The postoperative outcome was monitored; patients in ASA classes IV and V were admitted into intensive care unit after surgery. Final diagnosis and postoperative treatment was dependent on the operative findings and histopathological confirmation. Those found to be tuberculous were started on anti tuberculosis therapy according to the Tanzania National Tuberculosis and Leprosy Programme (NTLP). The anti tuberculosis therapy given included Isoniazid, Rifampicin, Pyrazinamide, Ethambutol and Streptomycin. Data on each patient were entered into a pro forma prepared for the study. The study variables included

\section{Table 1 American society of anesthetists (ASA)}

\section{classification}

\begin{tabular}{ll}
$\begin{array}{l}\text { ASA } \\
\text { class }\end{array}$ & Description \\
\hline I & Healthy individual with no systemic disease \\
III & $\begin{array}{l}\text { Mild systemic disease not limiting activity } \\
\text { Severe systemic disease that limits activity but is not } \\
\text { incapacitating }\end{array}$ \\
IV & $\begin{array}{l}\text { Incapacitating systemic disease which is constantly life } \\
\text { threatening }\end{array}$ \\
& $\begin{array}{l}\text { Moribund, not expected to survive } 24 \text { hours with or without } \\
\text { operation }\end{array}$ \\
\hline
\end{tabular}

socio-demographic (i.e. age and sex, level of education, occupation and area of residence), clinical presentation, HIV status, radiological findings, timing of surgical procedure, ASA classification, operative findings and surgical procedure performed. The variables studied in the postoperative period were postoperative complications, hospital stay and mortality. Patients were followed up for a period of twelve months or till death whichever is earlier.

\section{Definitions of terms}

Acute intestinal obstruction was considered if the patients had absolute constipation, nausea, vomiting and abdominal distension for 24-48 hours with radiological evidence supporting the clinical presentation.

Sub-acute intestinal obstruction was considered if the patients had relative constipation, nausea, vomiting and / or distension for more than 48 hours and the radiological findings were supporting the clinical findings.

Pulmonary tuberculosis was considered if the patient had sputum positive for acid-fast bacilli and / or X-ray was revealing pulmonary cavitatory lesion or calcified hilar lymph nodes.

Elective surgery that is scheduled in advance because it does not involve a medical emergency whereas an emergency surgery is one that must be performed without delay; the patient has no choice other than immediate surgery, if they do not want to risk permanent disability or death.

\section{Statistical data analysis}

The statistical analysis was performed using statistical package for social sciences (SPSS) version 17.0 for Windows (SPSS, Chicago IL, U.S.A). The mean \pm standard deviation (SD), median and ranges were calculated for continuous variables whereas proportions and frequency tables were used to summarize categorical variables. Chi-square $(x 2)$ test were used to test for the significance of association between the independent (predictor) and dependent (outcome) variables in the categorical variables. The level of significance was considered as $\mathrm{P}<0.05$. Multivariate logistic regression analysis was used to determine predictor variables that predict the postoperative complications, hospital stay and mortality.

\section{Ethical consideration}

Ethical approval to conduct the study was obtained from the CUHAS-Bugando/BMC joint institutional ethic review committee before the commencement of the study. Patients were required to sign a written informed consent for the study and for HIV testing.

\section{Results}

\section{Socio-demographic data}

During the study period, a total of 2643 patients were admitted to our centre and underwent laparotomy for 
various abdominal conditions. Of these 527 patients underwent laparotomy for bowel obstruction. Out of 527 patients, the underlying cause of obstruction was intestinal tuberculosis confirmed by histopathology in 129 patients. Of these, 11 patients were excluded from the study due failure to meet the inclusion criteria. Thus, 118 patients representing $22.4 \%$ of cases (i.e. 118 out of 527 patients) were enrolled into the study. Seventy-six (64.4\%) were males and $42(35.6 \%)$ females, with a male to female ratio of 1.8: 1 . The age of patients at presentation ranged from 11 to 67 years with a median age of 26 years. The peak age incidence was in the age group of 21-30 years accounting for $50.0 \%$ of cases (Figure 1). Eighty-eight $(74.6 \%)$ patients were aged 40 years and below. Most of patients, 91 (77.1\%) had either primary or no formal education and more than $75 \%$ of them were unemployed. The majority of patients, 86 (72.9\%) came from the rural areas located a considerable distance from the study area and more than $80 \%$ of them had no identifiable health insurance.

\section{Clinical presentation among patients with tuberculous bowel obstruction}

The duration of symptoms prior to admission varied between 4 days to 12 months with a median of 8 months. The majority of our patients, 68 (57.6\%) had symptoms of more than 6 months duration at the time of presentation. Out of 118 patients, 87 (73.7\%) were considered to have primary intestinal tuberculosis and the remaining 31 (26.3\%) had secondary intestinal tuberculosis (i.e. associated with pulmonary tuberculosis) with remarkable chest $\mathrm{x}$-rays, past history of pulmonary tuberculosis was positive in only 28 patients (23.7\%). Out of these, eight patients were on treatment with anti-tuberculous drugs while fourteen had already taken a complete course of anti-tubercular drugs. The remaining six patients were defaulters.
Sixty two (51.5\%) patients presented with acute intestinal obstruction, thirty-four $(28.8 \%)$ with sub-acute intestinal obstruction, sixteen (13.6\%) with signs of peritonism and six (5.1\%) with abdominal mass. Abdominal pain was the most common symptom and occurred in all cases (Table 2). In this study, twelve (10.2\%) patients had associated pre-morbid medical illness namely diabetes mellitus in 6 patients, hypertension in 4 patients, sickle cell disease and rheumatic heart in 1 patient each respectively. Twenty-five (21.2\%) patients were HIV positive. Of these, $8(32.0 \%)$ patients were known cases on anti-retroviral therapy (ARV) and the remaining $17(68.0 \%)$ patients were newly diagnosed patients. Out of 25 patients with HIV, 20 (80.0\%) patients were found to have risk factors for HIV infection. Of these, alcoholism [Odds Ratio 14.7, 95\% C.I. (7.2-19.3), $p=0.011$ ] and multiple sexual partners [Odds Ratio 9.5, 95\% C.I. (4.8-14.4), $\mathrm{p}=0.001$ ] were found to be independently and significantly associated with increased risk to HIV infection.

\section{Laboratory, radiological and histopathological investigations}

Complete Blood Count, Hemoglobin levels and ESR were done in all patients. More than three quarter of the patients had Hemoglobin levels less than $10.0 \mathrm{gm} / \mathrm{dl}$ and ESR in the first hour was found ranging between 40$140 \mathrm{~mm}$. Serological investigations for HIV infection revealed that $25(21.2 \%)$ patients were HIV positive. CD4 + count distribution among HIV positive patients ranged from 45 cells $/ \mu$ l to 688 cells $/ \mu$ l with the median CD $4+$ count of 225 cells $/ \mu$ l. A total of 7 HIV patients (28.0\%) had CD4+ count below 200 cells/ $\mu \mathrm{l}$ and the remaining patients $(72.0 \%)$ had CD4+ count of $\geq 200$ cells $/ \mu$ l. Serum electrolytes revealed hypokalaemia and hyponatraemia in 54 and 28 patients respectively. Serum albumin done in 78 patients revealed hypoalbuminaemia in 66 (84.6\%) patients. Plain abdominal $\mathrm{x}$-rays (erect/supine) done in all

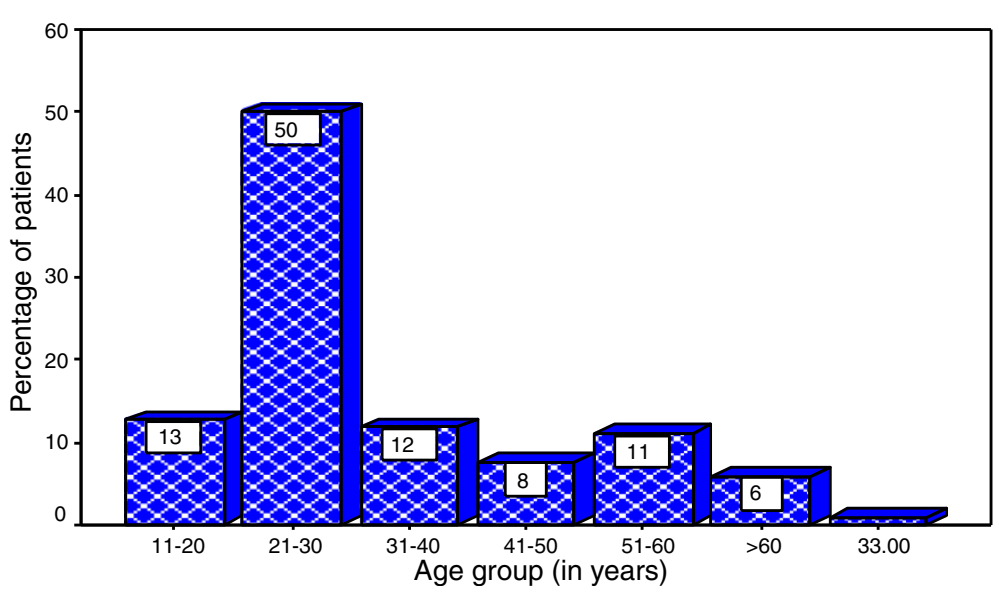

Figure 1 Distribution of patients according to age group. 


\begin{tabular}{|c|c|c|}
\hline Clinical presentation & Frequency & Percentage \\
\hline Abdominal pain & 118 & 100 \\
\hline Vomiting & 98 & 83.1 \\
\hline Constipation & 86 & 72.9 \\
\hline Weight loss & 80 & 67.8 \\
\hline Fever & 72 & 61.0 \\
\hline Abdominal distention & 62 & 52.5 \\
\hline Diarrhea/constipation & 25 & 21.2 \\
\hline Features of peritonism & 16 & 13.6 \\
\hline Abdominal tenderness & 82 & 69.5 \\
\hline Abdominal mass & 6 & 5.1 \\
\hline
\end{tabular}

patients revealed multiple dilated loops of bowel with significant air-fluid levels in erect films in 96 (81.4\%) patients. Free air under the right dome of diaphragm (pneumoperitonium) was seen in eight (6.8\%) patients. Radiography of the chest showed evidence of healed or active pulmonary tuberculosis in 28 (23.7\%) patients. Abdominal ultrasound revealed intraabdominal masses in six (5.1\%) patients. Barium studies done in 12 (10.2\%) revealed one or more of the features like narrowing of distal ileum and ileo-caecal region, matted small bowel. None of our patients had sigmoidoscopy, colonoscopy or Computered Tomography (CT) scan due to lack of these facilities at our centre. Histopathological examination revealed caseating granuloma in 88 cases of resected specimen of intestine only. In 32 patients these granuloma were found in mesenteric lymph nodes as well as intestine. In 8 patients, granulomata were found in parietal peritoneum and serosal tubercles.

\section{Preoperative anaesthetic assessment}

All patients were assessed pre-operatively using the American Society of Anesthetists (ASA) pre-operative grading. The majority of patients had ASA class II accounting for $61.0 \%$ of cases (Figure 2).

\section{Treatment modalities}

All the 118 patients had exploration of the abdomen. Sixty-nine (58.5\%) patients were operated on emergency bases while $49(41.5 \%)$ patients had an elective surgery. Operative findings of tuberculous intestinal obstruction are depicted in Table 3. The most common area of involvement was the ileo-caecal region in 68 (57.6\%) patients. This was followed by the terminal ileum and jejunum in $34(28.8 \%)$ and $12(10.2 \%)$ patients respectively. The colon was involved in $4(3.4 \%)$ patients. The main lesion causing obstruction was intestinal tuberculosis in the hypertrophic form in $86(72.9 \%)$ patients.
The right hemicolectomy with ileo-transverse anastomosis was the most common surgical procedure performed in $55.9 \%$ of the patients (Table 4). Postoperatively all the patients received antituberculous drugs for a period of one year.

\section{Treatment outcome Post-operative complications}

Forty-four (37.3\%) patients had 56 post-complications. Surgical site infection (SSI) was the most common postoperative complication accounting for $42.8 \%$ of cases (Table 5). In the present study, the rate of SSI was found to be significantly higher in HIV positive patients than in non HIV patients $(p=0.011)$. Also higher rate of SSI was observed among HIV patients with CD 4 count below 200 cells $/ \mu \mathrm{l}(p=0.021)$.

\section{Length of hospital stay}

The overall length of hospital stay (LOS) ranged from 1 to 64 days with a median of 24 days. The median LOS for non-survivors was 6 days (range 1-12 days). Patients who had post complications stayed longer in the hospital and this was statistically significant $(p=0.011)$.

\section{Mortality}

In this study, thirty-four patients died giving a mortality rate of $28.8 \%$. According to multivariate logistic regression analysis, co-existing medical illness $(\mathrm{OR}=4.5$, 95\% C.I. (2.58.9), $p=0.001)$, delayed presentation $(\mathrm{OR}=11.3,95 \% \mathrm{CI}$ (7.9- 18.4), $p=0.023)$, HIV positivity ( $\mathrm{OR}=5.9,95 \% \mathrm{CI}$ (3.1- 8.9), $p=0.002)$, low $\mathrm{CD} 4$ count $(<200$ cells $/ \mu \mathrm{l})(\mathrm{OR}=$ 7.0, 95\% CI (3.9-10.5), $p=0.000)$, high ASA class $(\mathrm{OR}=8.1$, 95\% CI (5.6-12.9), $p=0.014)$, surgical site infection (OR = $1.5,95 \% \mathrm{CI}(1.1-4.6), p=0.026)$ were the main predictors of mortality.

\section{Follow up of patients}

Of the survivors, seventy-eight (92.9\%) patients were discharged well and the remaining six (7.1\%) patients were discharged against medical advice. No patient among survivors in this study had permanent disabilities. Of the 84 survivors, thirty-four $(40.5 \%)$ patients were available for follow up at three to six months after discharge and the remaining 50 (59.5\%) patients were lost to follow up.

\section{Discussion}

In this review, the underlying cause of bowel obstruction was tuberculosis in $22.4 \%$ of patients, a figure which is comparable with $21.8 \%$ reported by Ali et al [22] in Pakistan. However, this figure is higher than that observed in many other studies [23-26]. These differences in the rate of tuberculous intestinal obstruction reflect differences in the prevalence and risk factors for developing complications of TB such as bowel obstruction 


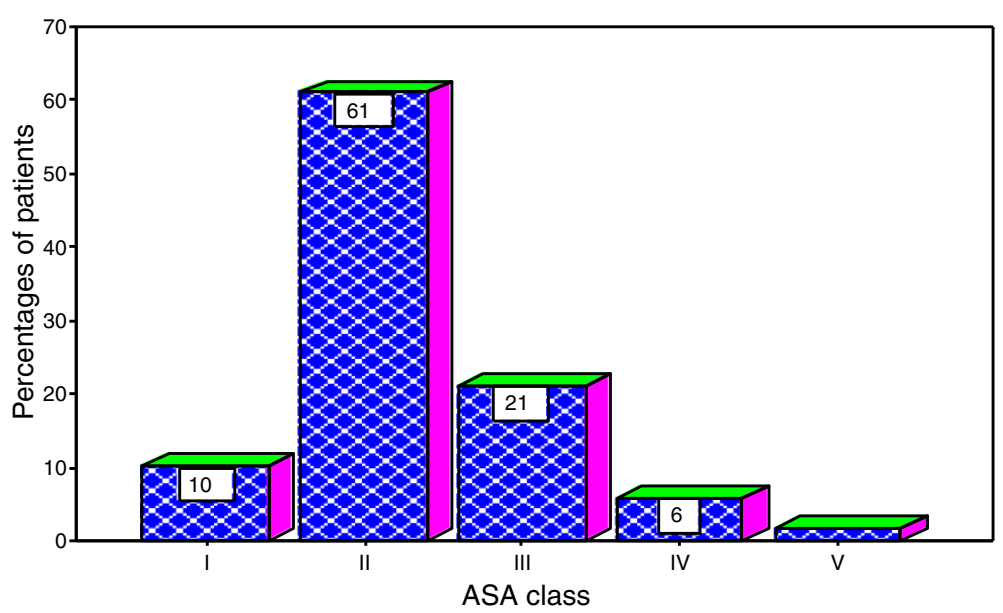

Figure 2 Distribution of patients according to ASA class.

among different study settings. The figures for the rate of tuberculous intestinal obstruction in our study may actually be an underestimate and the magnitude of the problem may not be apparent because of high number of patients excluded from this study.

This study showed that males were slightly more affected than females with a male to female ratio of 1.8:1 which is comparable to the global ratio of 1.5 to $2.1: 1$ [27]. Some workers report that the disease is more common in males in the western countries while in developing counties the females predominate [28]. We could not find in literature the reasons for this gender differences.

Intestinal tuberculosis, like tuberculosis elsewhere in the body affects the young people at the peak of their productive life [29]. This fact is reflected in our study as the highest age incidence of the patients was in the second and third decades of life and more than seventy percent of our patients were aged forty years and below. This is in accordance with the results of other workers $[16,30]$. The presentation of tuberculous intestinal obstruction in this age group has serious impacts on the national economy and production, as working and productive class of community is replaced by sick and ill individuals.

Intestinal obstruction resulting from tuberculous has been reported to be more prevalent in people with low socio-economic status [31]. This observation is reflected

Table 3 Distribution of patients according to operative findings $(\mathbf{N}=\mathbf{1 1 8})$

\begin{tabular}{lll}
\hline Operative findings & Frequency & Percentage \\
\hline Small bowel strictures (single/multiple) & 86 & 72.9 \\
Bands and adhesions & 20 & 16.9 \\
Bowel strictures and perforation & 6 & 5.1 \\
lleocaecal mass & 4 & 3.4 \\
Enlarged mesenteric lymph nodes & 2 & 1,7 \\
\hline
\end{tabular}

in our study where most of patients had either primary or no formal education and more than seventy-five percent of them were unemployed. The majority of patients in the present study came from the rural areas located a considerable distance from the study area and more than eighty percent of them had no identifiable health insurance. Similar observation was reported by others [10,31-35]. This observation has an implication on accessibility to health care facilities and awareness of the disease.

The clinical presentation of tuberculous intestinal obstruction in our patients is not different from those in other studies [35,36], with abdominal pain being common to all the patients. The clinical presentation of abdominal TB is usually non-specific $[37,38]$ and, therefore, often results in diagnostic delay and hence the development of complications such as intestinal obstruction [38]. In keeping with other studies $[33,35,36]$, the majority of our patients had symptoms of more than 6 months duration at the time of presentation. The reasons or late presentation in this study may be attributed to the fact that the diagnosis of intestinal TB in its initial stages is usually difficult due to vague and non-specific symptoms as a result patients remain undiagnosed for prolong periods, receiving symptomatic

Table 4 Distribution of patients according to type of surgical procedures performed

\begin{tabular}{lll}
\hline Type of surgical procedures & Frequency & Percentage \\
\hline $\begin{array}{l}\text { Right hemicolectomy with ileo-transverse } \\
\text { anastomosis }\end{array}$ & 66 & 55.9 \\
$\begin{array}{l}\text { Segmental bowel resection with end to } \\
\text { end anastomosis }\end{array}$ & 28 & 23.7 \\
Adhesion lysis & 20 & 16.9 \\
lleo-transverse bypass procedure & 2 & 1.7 \\
Ileostomy & 1 & 1.8 \\
Stricturoplasty & 1 & 1.8 \\
\hline
\end{tabular}


treatment and subsequently present late with complications such acute or sub-acute intestinal obstruction.

In our study, associated pulmonary tuberculosis was found in $23.7 \%$ of cases, a figure which is comparable with Baloch et al [39]. However, higher figures of associated pulmonary tuberculosis have been reported by others $[10,40]$. We could not find in literature, the reasons for these differences.

The presence of co-existing medical illness has been reported elsewhere to have an effect on the outcome of patients with tuberculous intestinal obstruction [41]. This is reflected in our study where patients with co-existing medical illness had significantly high mortality rate.

The prevalence of HIV infection in the present study was $21.2 \%$, a figure that is significantly higher than that in the general population in Tanzania (6.5\%) [42]. However, failure to detect HIV infection during window period and exclusion of some patients from the study may have underestimated the prevalence of HIV infection among these patients. High HIV seroprevalence among patients with tuberculous intestinal obstruction was also reported by Fee et al [43]. This difference in HIV seroprevalence among patients with tuberculous intestinal obstruction reflects differences in the overall prevalence for risk factors for HIV infection in general population from one country to another. High HIV seroprevalence in our study may be attributed to high percentage of the risk factors for HIV infection reported in the present study population.

The clinical picture of tuberculous intestinal obstruction may be complex when tuberculosis occurs with HIV infected patients [44]. HIV infection has been reported to increase the risk of surgical site infection and mortality [45]. In the present study, the rate of surgical site infections and mortality was found to be significantly higher in HIV positive patients than in non HIV patients. Also higher rate of SSI was observed among HIV patients with low CD 4 count $(<200$ cells/ $\mu \mathrm{l})$. Patients with AIDS usually have a more severe form of involvement than those who did not have AIDS $[37,46]$. However, the treatment of bowel obstruction

\begin{tabular}{|c|c|c|}
\hline Postoperative complications & Frequency & Percentage \\
\hline Surgical site infections & 24 & 42.9 \\
\hline Enterocutaneous fistula & 6 & 10.7 \\
\hline Wound dehiscence/ burst abdomen & 4 & 7.1 \\
\hline Paralytic ileus & 4 & 7.1 \\
\hline Intraabdominal abscess/ peritonitis & 3 & 5.4 \\
\hline Keloids & 3 & 5.4 \\
\hline Incisional hernia & 2 & 3.6 \\
\hline
\end{tabular}

due to tuberculosis in AIDS patients has been reported to be the same as in non-HIV infected patients [47] but multi-drug-resistant tuberculosis is more common in patients with AIDS [48].

Emergency surgical intervention is considered to be the standard treatment of choice for patients with tuberculous intestinal obstruction [36]. In keeping with other studies $[10,15,26,35,36]$, the majority of patients in this study underwent emergency surgical treatment. One of the many factors affecting the surgical outcome in patients with tuberculous intestinal obstruction is time interval between duration of onset of intestinal obstruction and surgical intervention [35,36]. In the present study, the majority of patients were operated more than 24 hours after the onset of illness. Similar observation was reported by other studies done elsewhere $[30,36]$. Delayed definitive surgery in the present study may be attributed to late presentation due to lack of accessibility to health care facilities, lack of awareness of the disease as a result some patients with tuberculous intestinal obstruction may decide to take medications in the prehospital period with hope that the symptoms will abate. It is also possible that some clinicians managing the patients initially may not have considered as a possible diagnosis. In resource-poor countries like Tanzania, difficulties in diagnosis of intestinal $\mathrm{TB}$, patient transfer, and inadequate medical treatment often result in delayed presentation to a hospital $[1,2,41]$.

In agreement with other studies $[16,36,49]$, the ileocaecal region was the most common site of the bowel affected. This is in sharp contrast to other authors who reported the terminal ileum as the most common site of involvement $[10,39]$. Many studies have been reported that the most common site of involvement of intestinal TB is the ileocaecal region, possibly because of the increased physiological stasis, increased rate of fluid and electrolyte absorption, minimal digestive activity and an abundance of lymphoid tissue at this site [9]. It has been shown that the $\mathrm{M}$ cells associated with Peyer's patches can phagocytes BCG bacilli [50]. The frequency of bowel involvement declines as one proceeds both proximally and distally from the ileocaecal region [9]. In this study, the main lesion causing obstruction was intestinal tuberculosis in the hypertrophic form which is in agreement with Nguyen [51] in Vietnam.

In the present study, the right hemicolectomy with ileo-transverse anastomosis was the most common surgical procedure performed in $55.9 \%$ of the patients. This was followed by segmental bowel resection with end to end anastomosis, release of adhesions, bypass surgery, ileostomy and stricturoplasty. Similar surgical treatment pattern was reported by other writers also $[15,52,53]$. In our study, stricturoplasty was performed in only one patient. This is in contrast to that reported by Akbar et al 
[36] who reported stricturoplasty as the most common surgical procedure performed. Stricturoplasty is a simple procedure originally described by Katariya and his colleagues in Chandigarh India in 1977 [54]. The procedure has become widely popular and is being practiced all over the world. It has also been tried in Crohn's disease, with the same usefulness [55]. The procedure is simple, quicker, less traumatic and applicable anywhere from pylorus to ileocaecal junction. The procedure can also be undertaken in active lesions [56]. However, this procedure was not popular in our study because the majority of our patients had very extensive local disease with long and multiple strictures and only option was either right hemicolectomy with ileo-transverse anastomosis or by segmental bowel resection with end to end anastomosis. Anti-tuberculous therapy was prescribed in all the tubercular patients postoperatively.

The presence of complications has an impact on the final outcome of patients presenting with tuberculous intestinal obstruction. In keeping with other studies $[32,36]$, surgical site infection was the most common postoperative complications in the present study. High rate of surgical site infection in the present study may be attributed to HIV seropositivity and low CD 4 count.

The overall median duration of hospital stay in the present study was 24 days which is higher than that reported by other authors $[20,25,35,36]$. This can be explained by the presence of large number of patients with postoperative complications in our study. However, due to the poor socio-economic conditions in Tanzania, the duration of inpatient stay for our patients may be longer than expected.

The overall mortality rate in this study was $22.7 \%$ and it was significantly associated with delayed presentation, HIV positivity, low CD 4 count, high ASA class and presence of complications. Addressing these factors responsible for high mortality in our patients is mandatory to be able to reduce mortality associated with this disease.

Self discharge by patient against medical advice is a recognized problem in our setting. Similarly, poor follow up visits after discharge from hospitals remain a cause for concern. These issues are often the results of poverty, long distance from the hospitals and ignorance. Delayed presentation, delayed histopathological confirmation of tuberculous bowel obstruction and the large number of loss to follow up were the major limitations in this study.

However, despite these limitations, the study has provided local data that can be utilized by health care providers to plan for preventive strategies as well as establishment of management guidelines for these patients. The challenges identified in the management of patients with tuberculous bowel obstruction in our environment need to be addressed, in order to deliver optimal care for these patients.

\section{Conclusion}

Bowel obstruction resulting from intestinal tuberculosis is one of the most common abdominal surgical emergencies in our environment and contributes significantly to high morbidity and mortality. Young age at presentation, delayed presentation, poverty and high morbidity and mortality are among the hallmarks of the disease in this region. A high index of suspicion, proper evaluation and therapeutic trial in suspected patients is essential for an early diagnosis and timely definitive treatment, in order to decrease the morbidity and mortality associated with this disease. Factors that were found to be associated with high morbidity and mortality in this study need to be addressed.

\section{Competing interests}

The authors declare that they have no competing interests. The study had no external funding. Operational costs were met by authors.

\section{Authors' contributions}

PLC participated in study design, literature search, data analysis, manuscript writing, editing and submission of the manuscript. MDM, SEM, PR, HJ and JBM participated in data analysis, manuscript writing \& editing. All the authors read and approved the final manuscript.

\section{Acknowledgement}

The authors are grateful to all who participated in the preparation of this manuscript. Special thanks go to our research assistants for data collection.

\section{Author details}

'Department of Surgery, Catholic University of Health and Allied SciencesBugando, Mwanza, Tanzania. ${ }^{2}$ Department of Surgery, Muhimbili University of Health and Allied Sciences, Dar Es Salaam, Tanzania. ${ }^{3}$ Department of Microbiology \& Immunology, Catholic University of Health and Allied Sciences-Bugando, Mwanza, Tanzania. ${ }^{4}$ Department of Pathology, Catholic University of Health and Allied Sciences-Bugando, Mwanza, Tanzania. ${ }^{5}$ Department of Internal Medicine, Catholic University of Health and Allied Sciences-Bugando, Mwanza, Tanzania.

Received: 27 January 2013 Accepted: 8 March 2013 Published: 16 March 2013

\section{References}

1. Lonnroth K, Raviglion M: Global epidemiology of tuberculosis: prospects for control. Semin Respir Crit Care Med 2008, 29:481.

2. Dolin PJ, Raviglione MC, Kochi A: Global tuberculosis incidence and mortality during 1990-2000. Bull World Health Organ 1994, 72:213-220.

3. Tan K-K, Chen K, Sim R: The spectrum of abdominal tuberculosis in a developed country: a single institution's experience over 7 years. J Gastrointest Surg 2009, 13:142-147.

4. Sharp JF, Goldman M: Abdominal Tuberculosis in East Birmingham, a 16 years study. Postgrad Med J 2002, 63:539-542.

5. Butt T, Karamat KA, Ahmad RN, Mahmood A: Advances in diagnosis of tuberculosis. Pak J Pathol. 2001, 12:1-3.

6. WHO: Global Tuberculosis control. Geneva: World Health Organization; 2008.

7. Ducati RG, Ruffino NA, Basso LA, Santos DS: The resumption of consumption - a review on tuberculosis. Mem Inst Oswaldo Cruz 2006, 101:697-714.

8. Khan MR, Khan IR, Pal KNM: Diagnostic issues in abdominal tuberculosis. J Pak Med Assoc 2001, 51:138-140.

9. Sharma MP, Bhatia V: Abdominal tuberculosis. Indian J Med Res 2004 120:305-315.

10. Shaikh MS, Dholia KR, Jalbani MA: Prevalence of intestinal tuberculosis in cases of acute abdomen. Pakistan J Surg 2007, 23:52-56.

11. Engin G, Balk E: Imaging findings of Intestinal Tuberculosis. J Comput Assist Tomogr 2005, 29:37-41.

12. Rita S: Diagnosis of abdominal tuberculosis. Role of imaging. J Ind Acad Clin Med 2001, 2:103-104. 
13. Ahmed M, Mainghal MA: Pattern of mechanical intestinal obstruction in adults. J Coll Physicians Surg Pak 1999, 9:441-443.

14. Gondal KM, Khan AFA: Changing pattern of abdominal tuberculosis. Pak J Surg 1995, 11:109-113.

15. Shaikh MS, Ramdholia K, Jalbani MA, Shaikh SA: Prevalence of intestinal tuberculosis in cases of acute abdomen. Pak J Surg 2007, 23:52-56.

16. Rajpoot MJ, Memon AS, Rani S, Memon AH: Clinicopathological profile and surgical management outcomes in patients suffering from intestinal tuberculosis. J Liaqaut Uni Med Health Sci 2005, 4:113-118.

17. Anuradha B, Aparan S, Hari SPV, Vijaya LV, Akbar Y, Suman LG: Prevalence of drug resistance under the DOTs Strategy in Hyderabad South India, 2001-2003. Int J Tuberc Lung Dis 2006, 10:58-62.

18. Wolters U, Wolf T, Stutzer H, Schroder T: ASA classification and perioperative variables as predictors of postoperative outcome. British Journal of Anesthesia 1996, 77:217-222.

19. Lyamuya EF, Aboud S, Urassa WK, Sufi J, Mbwana J, Ndungulile F, Massambu C: Evaluation of rapid HIV assays and development of national rapid HIV test algorithms in Dar es salaam, Tanzania BMC infectious diseases 2009, 9:19.

20. Ahmed EHG, Nassar AS, Ginawi l: Screening for tuberculosis and its histological pattern in patients with enlarged lymph node. Pathol Res Int 2011, 417635:4. doi:10.4061/2011/417635.

21. Soylu A, Ince AT, Polat H, Yasar N, Ciltas A, Ozkara S, Tasci Al: Peritoneal tuberculosis and granulomatous hepatitis secondary to treatment of bladder cancer with Bacillus Calmette-Guérin. Ann Clin Microbiol Antimicrob 2009, 8:12. doi:10.1186/1476-0711-8-12.

22. Ali N, Hussein M, Israr M: Tuberculosis as a cause of small bowel obstruction in adults. Gomal Journal of Medical Sciences 2011, 9:233-235.

23. Hasnain SQ, Ahmad M: Intestinal obstruction in adults at Aga khan university hospital. J Pak Assoc 1994, 44:143-145.

24. Atiq A: Aetiological aspects of dynamic intestinal obstruction: Mayo Hospital experience. Pak J Surg 1996, 12:118-119.

25. Manzoor A, Muhammad AM: Pattern of mechanical intestinal obstruction in adults. J Col Physicians Surg 1999, 9:441-443.

26. Abdudllah SI, Parwaiz I: Tuberculosis: a common cause of intestinal obstruction. Pak J Surg 1998, 14:73-75.

27. Vinod KD, Anna J: Sex gender and tuberculosis. Lancet 1999, 353:1000-1001.

28. Homan WP, Grofe WR, Dineem P: A 44-year experience with tuberculous enterocolitis. World J Surg 1977, 2:45-50.

29. Khan SM, Khan KM, Khan AS, Jehanzeb M, Jan WA, Khan M, Ali U: Presentation of abdominal tuberculosis in NWFP and its correlation with operative findings. J Postgrad Med Inst 2005, 19:286-291.

30. Gondal SH, Gulshan S, Naseeb U: Intestinal Tuberculosis as an abdominal emergency. Pak Postgrad Med J 2000, 11:103-105.

31. Gomez JE, McKinney JD: Tuberculosis persistence, latency and drug tolerance. Tuberculosis 2004, 84:29-44.

32. Niaz K, Ashraf M: Intestinal tuberculosis; Diagnostic dilemma. Professional Med J 2010, 17:532-537.

33. Schmidt PJ, Petrie B, Thompson JE: Abdominal tuberculosis; the surgical prospective. Am J Surg 1996, 62:856-858.

34. Baloch N, Tufail M, Durrani K, Mahmood A: Abdominal tuberculosis: a varied presentation. Pakistan J Med Res 1993, 32:259-262.

35. Iqbal $T$, Khan A, lqbal A, Tahir F: Obstruction due to intestinal tuberculosis strictureplasty versus resection anastomosis. Pak J Surg 2008, 24:177-181

36. Akbar M, Islam F, Haider IZ, Naveed D, Akbar I, Khattak I, Akbar K, Zafar A: Surgical management of tuberculous small bowel obstruction. J Ayub Med Coll Abbottabad 2010, 22:171-175.

37. Lazarus AA, Thilagar B: Abdominal tuberculosis. Dis Mon 2007, 53:32-38

38. Rasheed S, Zinicola R, Watson D, Bajwa A, McDonald PJ: Intra-abdominal and gastrointestinal tuberculosis. Colorectal Dis 2007, 9:773-783.

39. Baloch NA, Baloch MA, Baloch AF: A study of 86 cases of abdominal tuberculosis. Journal of Surgery Pakistan (International) 2008, 13:30-32.

40. Boukthir S, Murad SM: Abdominal Tuberculosis in children. Acta Gastroenterol Belg 2004, 67:245-249.

41. Hu ML, Lee CH, Kuo CM, Huang CC, Tai WC, Chang KC, Lee CM, Chuah SK. Abdominal tuberculosis: analysis of clinical features and outcome of adult patients in southern Taiwan. Chang Gung Med J 2009, 32:510-515.

42. Urassa M, Isingo R, Kumogola Y, Mwidunda P, Helelwa M, Changulucha J, Mngara J, Zaba B, Calleja T, Slaymaker E: Effect of PMTCT availability on choice of ANC in Mwanza and Magu districts and its impact on HIV sentinel surveillance in Tanzania. Report of ANC surveillance Mwanza and Magu Districts; 2007

43. Fee MJ, Oo MM, Gabayan AE, Radin DR, Barnes PF: Abdominal tuberculosis in patients infected with the human immunodeficiency virus. Clin Infect Dis 1995, 20:938-944

44. Iliyasu Z, Babashani M: Prevalence and predictors of tuberculous coinfection among HIV seropositive patients attending the Aminu Kano Teaching Hospital, northern Nigeria. J Epidemiol 2009, 19:81-87.

45. Mawalla BM, Mshana SE, Chalya PL, Imirzalioglu C, Mahalu W: Predictors of surgical site infections among patients undergoing major surgery at Bugando Medical Centre in Northwestern Tanzania. BMC Surg 2011, 11:21.

46. Balthazar EJ, Gordon R, Hulnick D: lleocecal tuberculosis: CT and radiographic evaluation. AJR Am J Roentgenol 1990, 154:499-503.

47. Watters DAK: Surgery for tuberculosis before and after HIV infection: a tropical perspective. Br J Surg 1997, 84:8-14.

48. Iseman MD: Treatment of multidrug resistant tuberculosis. N Engl Jt Med 1993, 329:784-791.

49. Wadhwa N, Agarwal S, Mishra K: Reappraisal of abdominal tuberculosis. J Ind Med Assoc 2004, 102:31-42.

50. Paustian FF: Tuberculosis of the intestine. In Bockus HL, editor. Gastroenterology, Volume 11. 2nd edition. Philadelphia: W.B. Saunders Co; 1964:p. 311.

51. Nguyen VH: Intestinal obstruction due to tuberculosis. Asian J Surg 2002, 25:145-148.

52. Sial K, Baloch Q: Presentation and surgical management of Abdominal Tuberculosis. Med Channel 2004, 10:20-22.

53. Tariq N: Abdominal Tuberculosis. The surgical audit of its presentation. Pak J Surg 1993, 13:82-88.

54. Katariya RN, Sood S, Rao PG, Rao PLNG: Stricturoplasty for tubercular stricture of the gastrointestinal tract. Br J Surg 1977, 64:494-496.

55. Prichard TJ, Schoetz DJ, Caushaj FP, Roberts LP, Marry JJ: Strictroplasty of small bowel in patients with Crohn's Disease. Arch Surg 1990, 125:715-717.

56. Pujari BD: Modified surgical procedures in intestinal tuberculosis. $\mathrm{Br} J$ Surg 1979, 66:180-184.

doi:10.1186/1749-7922-8-12

Cite this article as: Chalya et al: Tuberculous bowel obstruction at a university teaching hospital in Northwestern Tanzania: a surgical experience with 118 cases. World Journal of Emergency Surgery 2013 8:12.

\section{Submit your next manuscript to BioMed Central and take full advantage of:}

- Convenient online submission

- Thorough peer review

- No space constraints or color figure charges

- Immediate publication on acceptance

- Inclusion in PubMed, CAS, Scopus and Google Scholar

- Research which is freely available for redistribution

Submit your manuscript at www.biomedcentral.com/submit
C BioMed Central 